\title{
SOIL MOISTURE RETRIEVAL USING CONVOLUTIONAL NEURAL NETWORKS: APPLICATION TO PASSIVE MICROWAVE REMOTE SENSING
}

\author{
Zhongzheng $\mathrm{Hu}^{1}$, Linlin $\mathrm{Xu}^{1 *}$, Bowen $\mathrm{Yu}^{1}$, \\ ${ }^{1}$ CUGB, School Of land Science And Technology, Beijing, China \\ hzz_cugb@163.com, beyond13031@126.com,594539169@qq.com
}

Commission III, WG III/2

KEY WORDS: Soil Moisture Retrieval, Microwave Remote Sensing, Deep Learning, Convolutional Neural networks, GPU

\begin{abstract}
:
A empirical model is established to analyse the daily retrieval of soil moisture from passive microwave remote sensing using convolutional neural networks (CNN). Soil moisture plays an important role in the water cycle. However, with the rapidly increasing of the acquiring technology for remotely sensed data, it's a hard task for remote sensing practitioners to find a fast and convenient model to deal with the massive data. In this paper, the AMSR-E brightness temperatures are used to train CNN for the prediction of the European centre for medium-range weather forecasts (ECMWF) model. Compared with the classical inversion methods, the deep learning-based method is more suitable for global soil moisture retrieval. It is very well supported by graphics processing unit (GPU) acceleration, which can meet the demand of massive data inversion. Once the model trained, a global soil moisture map can be predicted in less than 10 seconds. What's more, the method of soil moisture retrieval based on deep learning can learn the complex texture features from the big remote sensing data. In this experiment, the results demonstrates that the CNN deployed to retrieve global soil moisture can achieve a better performance than the support vector regression (SVR) for soil moisture retrieval.
\end{abstract}

\section{INTRODUCTION}

A methodology to retrieve soil moisture from passive microwave remote sensing with convolutional neural networks $(\mathrm{CNN})$ is presented. Soil moisture is a very important variable of water cycle, which can be used to predict the drought or crop yield in agriculture. Simultaneously, in some ongoing engineering construction (like housing, railway, road and bridge etc.), it also plays an important role. However, with the rapidly increasing of the acquiring technology for remotely sensed data, there are lots of different sensors for soil moisture retrieval, and the remotely sensed soil moisture products include:

(a) The national aeronautics and space administration (NASA) soil moisture product (Tuttle et al., 2014);

(b) The land parameter retrieval model (LPRM) soil moisture product (Dall'Amico et al., 2009);

(c) The Japan aerospace exploration agency (JAXA) soil moisture product (Cui et al., 2016);

(d) The JAXA AMSR2 soil moisture product (Lu et al., 2017);

(e) The soil moisture and ocean salinity soil moisture (SMOS) product (Kerr et al., 2012);

(f) The advanced scatterometer (ASCAT) soil moisture product (Wagner et al., 2013);

(g) The essential climate variable (ECV) soil moisture product (Wang et al., 2016);

(h) The European centre for medium-range weather forecasts (ECMWF) soil moisture product (Buizza et al., 1999).

The first three products $(\mathrm{a}-\mathrm{c})$ are derived from the advanced microwave scanning radiometer - earth observing system (AMSR-E) (Zeng et al., 2015), and other five products (d-h) are retrieved from other different sensors.

\footnotetext{
* Corresponding author
}

Corresponding to this, a lot of traditional soil moisture retrieval algorithms were proposed, such as SMOS Level 2 and Level 3 operational algorithms, SMAP Level 2 operational algorithm, the dual channel algorithm (DCA), land parameter retrieval model (LPRM), multi-orbit retrievals of soil moisture and optical depth (MT-DCA), and various other retrieval algorithms based on neural networks or local regressions (Wigneron et al., 2017). There are many methods to retrieve soil moisture, but they cannot meet the demands of remote sensing big data. So, it's a hard task for remote sensing practitioners to find a fast and convenient model to retrieve soil moisture with the massive data. In this paper, a deep learning based empirical model is well used for soil moisture retrieval.

Presently, a suitable method to inverse soil moisture by passive microwave remote sensing is deep learning in the context of remote sensing big data. Deep learning is a class of machine learning algorithm. It is capable of "learning features" from the data at each level and processing the complex input data and learning tasks. With the rapid increasing of remote sensing data, traditional retrieval methods, either empirical or physical models, cannot satisfy the requirement of the high complexity of the data and the non-linearity of retrieval problems (Ali et al., 2015). Therefore, it is vital to develop the advanced method for inversion of soil moisture based on the deep learning technique. Compared with the classical inversion methods, the deep learning-based method is more suitable for soil moisture retrieval.

Support vector regression (SVR) is a popular approach in the field of geo-/bio-physical parameter retrieval, which however only has the good intrinsic generalization ability and the robustness to noise in the case of limited availability of the reference samples (Durbha et al., 2007). In addition, traditional 
models is not flexible enough to learn more about feature information. Therefore, it is of great advantage to investigate deep learning based soil moisture retrieval approach in comparison with classical algorithms.

What's more, the method of soil moisture retrieval based on deep learning is very well supported by graphics processing unit (GPU) acceleration, which can meet the demand of massive data inversion. However, traditional retrieval algorithms don't support GPU parallel acceleration, which will waste more resources in the inversion of soil moisture. Once trained, the deep learning model is a very efficient method to retrieve soil moisture (Rodríguez-Fernández> et al ., 2015). As a result, it is of great value to study using GPU-accelerated soil moisture inversion based on the deep learning method.

For the first time, this paper uses deep learning in the inversion of soil moisture content, which can learn the complex features from the big remote sensing data better and retrieve soil moisture in real time compared with classical algorithms. The $\mathrm{CNN}$ algorithm used in this research is composed of three pairs of convolution layers and pooling layers with one fully connected layer on top, whose activation function of the top layer is changed from softmax loss layer to Euclidean loss layer. The AMSR-E brightness temperature images are used as input, and the soil moisture value gained from ECMWF model which is considered the most accurate value of soil moisture content is used as ground truth. In this experiment, one month's global data which include 30 pairs of images is used to train the deep learning model, and then it is used to predict the next month's data for soil moisture maps. By comparing the root-meansquare error (RMSE) and the R-square $\left(R^{\wedge} 2\right)$ with SVR, the experiment demonstrates the deep learning method for soil moisture retrieval can achieve better learn the complex relationship between the observations and the ground truth and achieve better generalization performance compared with traditional retrieval algorithms.

This paper is organized as follows. Section 2 provides the datasets used in this study. Section 3 describes the methodology employed to establish CNN and train regression model for soil moisture retrieval. Section 4 discusses the experiment and the results of soil moisture retrieval using $\mathrm{CNN}$ model. Section 5 summarizes the conclusions of the study.

\section{DATA}

The soil moisture retrieval is performed using data from a passive microwave sensor, AMSR-E, as well as modelled soil moisture reanalysis from ERA-interim in the ECMWF model which is a global atmospheric reanalysis from 1979, continuously updated in real time. The study focuses on one month's global data in July, 2011. All data are projected onto an equal area grid with a $0.25^{\circ}$ resolution at the equator.

\begin{tabular}{ccccc}
\hline Method & Data & Training & Validation & Testing \\
\hline \multirow{2}{*}{ CNN } & AMSR-E & $30 \times 30000$ & $30 \times 5000$ & $1 \times 50000$ \\
& ECMWF & $30 \times 30000$ & $30 \times 5000$ & $1 \times 50000$ \\
& AMSR-E & $30 \times 30000$ & $30 \times 5000$ & $1 \times 50000$ \\
SVR & ECMWF & $30 \times 30000$ & $30 \times 5000$ & $1 \times 50000$
\end{tabular}

Table 1. The samples used in this experiment.
The data used in this paper are as above (Table 1), all the samples are patches of size $9 \times 9$. The images from July $1^{\text {st }}$ to $30^{\text {th }}$ are used as training and validation sets, and there are 35000 image patches every day. 30000 pairs image patches are used to train CNN model, and the rest are validation sets. For evaluating the quality of the model, the 50000 image patches from July $31^{\text {th }}$ are used to predict the global soil moisture map.

\subsection{Advanced Microwave Scanning Radiometer-Earth Obs- erving System (AMSR-E)}

The AMSR-E was a passive microwave sensor which observes brightness temperatures at $6.9,10.7,18.7,23,37$ and $89 \mathrm{GHz}$ at vertical and horizontal polarizations (Kolassa et al., 2017). The sensor was flown aboard the aqua satellite between 2002 to 2011 with a revisit time of 1-2 days. In this paper, the $6.9 \mathrm{GHz}$ and $10.7 \mathrm{GHz}$ channels were used, because they have the strongest sensitivity to soil moisture. In order to use the AMSRE data as input to the CNNs, a pre-processing step is necessary to eliminate outliers which will have a bad influence on the precision of training.

\subsection{ERA-interim}

Modelled soil moisture fields from ERA-interim have been used to calibrate the retrieval and to assess the retrieval performance. The soil moisture value gained from ECMWF model which is considered the most accurate value of soil moisture content is used as ground truth. The ECMWF products used in this work are operational integrated forecasting system (IFS) models with the "hydrology-improved tiled ECMWF scheme for surface exchanges over land" (H-TESSEL) (Slingo, 1987). In this paper, the data is provided on a regular grid of $0.25^{\circ}$ spatial resolution, and there are 12 time steps per day and location. The average of these 12 time steps has been used here to get a daily modelled soil moisture estimate. In this study, only the upper layer soil moisture, representing a depth of $7 \mathrm{~cm}$, has been used, which is a few centi-meters deeper than the typical penetration depth of the microwave satellite data.

\section{METHODOLOGY}

In this paper, a supervised $\mathrm{CNN}$ model is used as a feature extractor and a regression model to retrieve the daily global soil moisture from the brightness temperature. The processing scheme is shown in Figure 1. It is composed of three major steps: preprocessing of the images, training of the CNN model, and prediction in new brightness temperature using the trained CNN model. Only the first steps will be described, because the prediction follows the same as the testing.

\subsection{Preprocessing of the Images}

All the images employed to train $\mathrm{CNN}$ model need to be reprocessed, and the major steps includes image registration, spatial interpolation, and normalization. Because the one day's data cannot overlap the whole world, it was a vital step to registrate 1-2 day's AMSR-E data for obtaining the average images. In addition, the brightness temperature data and soil moisture truth value from ECMWF had different resolution. In this paper, the soil moisture truth value images grid with a $0.5^{\circ}$ resolution have been projected onto an equal area grid with a $0.25^{\circ}$ resolution the same as brightness temperature data. Then the inputs and outputs are normalized to $[0,1]$. After 
reprocessing, many patch images of size $9 \times 9$ were generated, the number of which is given in Table 1 .

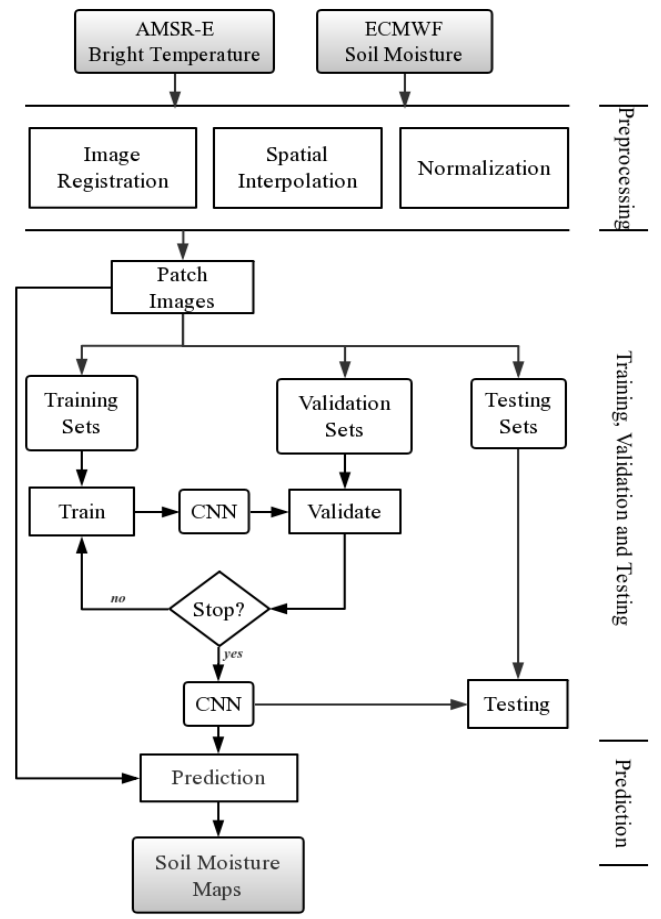

Figure 1. The flowchart of the major steps of the algorithm.

\subsection{Structure of CNN}

Multilayer CNNs are a very efficient method to image classification. In this paper, the CNNs is employed to find a function linking a set of input data to SM. It can satisfy the requirement of the high complexity of the data and the non-linearity of retrieval problems.

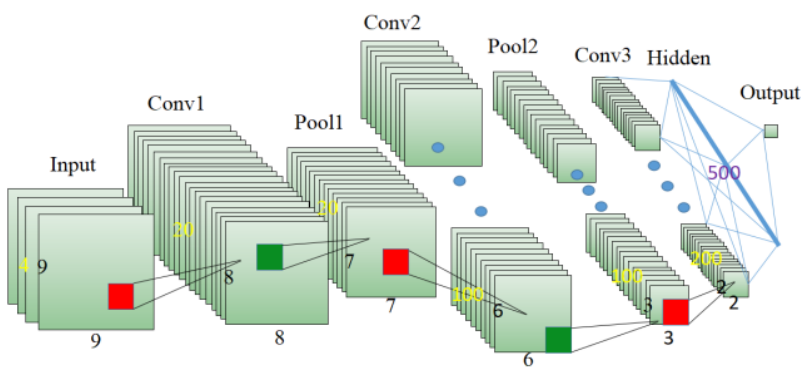

Figure 2. The structure of the proposed CNN model.

The CNNs used in this study has three pairs of convolution layers and pooling layers with one fully connected layer on top, whose activation function of the top layer is changed from softmax loss layer to Euclidean loss layer, as shown in the picture (Figure. 2). The first convolutional layer has 20 filters of width and height set to two. Inputs to the $\mathrm{CNNs}$ are image patches of size $9 \times 9 \times 4$; thus, the size of the filter is $(2,2,4)$. The output of the first convolution is the array of size $(9-2+1,9-2+1$, $20)$, that is $(8,8,20)$. The first convolution layer is followed by a max pooling layer of pooling size $2 \times 2$ and stride 1 . After the transfer of max pooling layer, the size of the image feature maps changed to $(7,7,20)$. The second convolution layer contains 100 filters of size $(2,2,20)$. It outputs convolved image of size
(7-2+1,7-2+1,100). The following pooling layer is a array which size is $2 \times 2$ and stride 2 . Its feature map is $(3,3,100)$. The third convolution layer is composed of 200 filters that size is $2 \times 2$ and stride 1 . Its outputs feature map is $(2,2,200)$. In this paper, the hidden layer (the first fully connected layer shown as Fig. 2) of dimension $500 \times 1$ follows the third convolution instead of the max polling layer. The second fully connected layer takes outputs only one single value, which is the truth value of soil moisture. By modifying the activation function of the top layer, the classification CNN model is transferred to $\mathrm{CNN}$ regression model. In this study, the top layer is changed from softmax loss layer to Euclidean loss layer.

\section{DISCUSSION}

In this paper, the follow images show the result of the soil moisture retrieval using CNN model and using SVR.

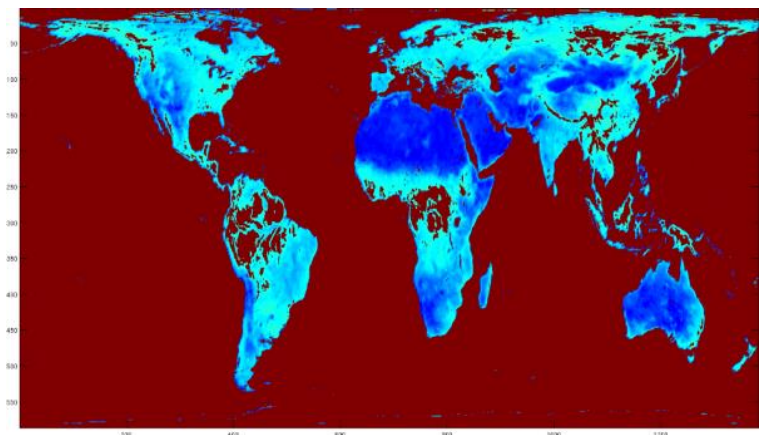

Figure 3. The soil moisture prediction using the CNN model.

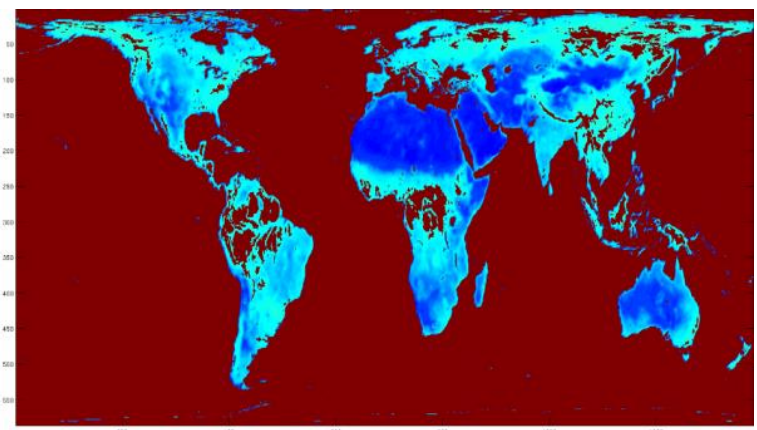

Figure 4. The soil moisture prediction using the SVR model.

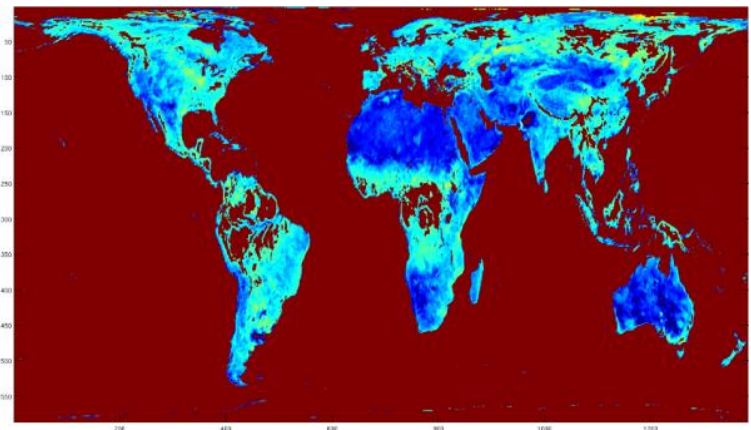

Figure 5. The soil moisture ground truth from AMSR-E.

As shown in Figure 3-5, the prediction map using the CNN model is closer to the truth label of global soil moisture (Figure. 5). In some parts of southern and northern Africa, as well as 
Australia, the CNN model performs better against the SVR model. The results suggest that the CNN (Figure 3) deployed to retrieve global soil moisture can achieve a better performance than the SVR (Figure 4) for soil moisture retrieval.

\begin{tabular}{ccc}
\hline & $\mathrm{R}^{\wedge} 2$ & RMSE \\
\hline CNN & 0.6512 & 0.1272 \\
SVR & 0.5598 & 0.1817 \\
\hline
\end{tabular}

Table 2. The RMSE and $\mathrm{R}^{\wedge} 2$ of CNN and SVR.

In this study, as the above Table 2 shows, by comparing the root-mean-square error (RMSE) and the R-square $\left(\mathrm{R}^{\wedge} 2\right)$, the CNN model have a $\mathrm{R}^{\wedge} 2$ (0.6512) higher than the SVR (0.5598), and the RMSE (0.1272) is less than the latter (0.1817). What's more, the prediction using the $\mathrm{CNN}$ model takes less time than the SVR model in global soil moisture retrieval.

\section{CONCLUSION}

In this paper, a CNN has been employed to AMSR-E brightness temperatures images to retrieve the global soil moisture daily. The CNN used took the image patches of brightness temperature data as input and output soil moisture value directly. When compared to classical SVR approach, the CNN method achieved soil moisture results that are closer to the ground truth map.

The training on 31 images took about 2 hours, the prediction of soil moisture on one image took less than 10 seconds using an Nvidia GTX 1080Ti graphics card. With more powerful or more quantity graphics cards, the time spent on training and predicting will be reduced largely. Once the model between the brightness temperature and soil moisture trained, the soil moisture retrieval can run in parallel and multiple GPUs easily. However, the prediction of soil moisture on one image using SVR took more than two minutes. Therefore, comparing with traditional regression approaches, $\mathrm{CNN}$ had a great advantage on both prediction accuracy and computational cost for the retrieval of soil moisture from big remote sensing data.

\section{ACKNOWLEDGEMENTS}

This work was supported by the National Natural Science Foundation of China Grant 41501410.

\section{REFERENCES}

Zeng, J., Li, Z., Chen, Q., Bi, H., Qiu, J., \& Zou, P. (2015). Evaluation of remotely sensed and reanalysis soil moisture products over the tibetan plateau using in-situ observations. Remote Sensing of Environment, 163, 91-110.

Tuttle, S. E., \& Salvucci, G. D. (2014). A new approach for validating satellite estimates of soil moisture using large-scale precipitation: comparing amsr-e products. Remote Sensing of Environment, 142(3), 207-222.

Dall'Amico, J. T., Loew, A., Schlenz, F., \& Mauser, W. (2009). SMOS rehearsal campaign 2008: radiometer data analysis and soil moisture retrieval using the LPRM. Earth Observation and Water Cycle conferenceEarth Observation and Water Cycle conference.
Cui, H., Jiang, L., Du, J., Wang, G., \& Lu, Z. (2016). Assessment of QP model based two channel algorithm with JAXA, LPRM soil moisture products over Genhe area in China. Geoscience and Remote Sensing Symposium (pp.1663-1666). IEEE.

Lu, Z., Chai, L., Zhang, T., Cui, H., \& Li, W. (2017). Evaluation of amsr 2 retrievals using observation of soil moisture network on the upper and middle reaches of heihe river basin. Remote Sensing Technology \& Application .

Kerr, Y. H., Waldteufel, P., Richaume, P., Wigneron, J. P., Ferrazzoli, P., \& Mahmoodi, A., et al. (2012). The smos soil moisture retrieval algorithm. IEEE Transactions on Geoscience \& Remote Sensing, 50(5), 1384-1403.

Wagner, W., Hahn, S., Kidd, R., Melzer, T., Bartalis, Z., \& Hasenauer, S., et al. (2013). The ascat soil moisture product: a review of its specifications, validation results, and emerging applications. Meteorologische Zeitschrift, 22(1), 5-33.

Wang, S., Mo, X., Liu, S., Lin, Z., \& Hu, S. (2016). Validation and trend analysis of ecv soil moisture data on cropland in north china plain during 1981-2010. International Journal of Applied Earth Observation \& Geoinformation, 48(48), 110-121.

Buizza, R., Milleer, M., \& Palmer, T. N. (1999). Stochastic representation of model uncertainties in the ecmwf ensemble prediction system. Quarterly Journal of the Royal Meteorological Society, 125(560), 2887-2908.

Wigneron, J. P., Jackson, T. J., O'Neill, P., Lannoy, G. D., Rosnay, P. D., \& Walker, J. P., et al. (2017). Modelling the passive microwave signature from land surfaces: a review of recent results and application to the 1-band smos \& smap soil moisture retrieval algorithms. Remote Sensing of Environment, $192,238-262$.

Ali, I., Greifeneder, F., Stamenkovic, J., Neumann, M., \& Notarnicola, C. (2015). Review of machine learning approaches for biomass and soil moisture retrievals from remote sensing data. Remote Sensing, 7(12), 221-236.

Durbha, S. S., King, R. L., \& Younan, N. H. (2007). Support vector machines regression for retrieval of leaf area index from multiangle imaging spectroradiometer. Remote Sensing of Environment, 107(1), 348-361.

Rodríguez-Fernández>, N. J., Aires, F., Richaume, P., Kerr, Y. H., Prigent, C., \& Kolassa, J., et al. (2015). Soil moisture retrieval using neural networks: application to smos. IEEE Transactions on Geoscience \& Remote Sensing, 53(11), 59916007.

Kolassa, J., Gentine, P., Prigent, C., Aires, F., \& Alemohammad, S. H. (2017). Soil moisture retrieval from amsr-e and ascat microwave observation synergy. part 2: product evaluation. Remote Sensing of Environment, 195, 202-217.

Slingo, J. M. (1987). The development and verification of a cloud prediction scheme in the ecmwf model.

Quart.j.roy.meteor.soc, 113 . 